Supporting Information

\title{
Electrical patterning of graphene circuitry by hydrogenation for transparent and flexible devices
}

Jangyup Son $\uparrow$, Jongin Cha and Jongill Hong*

Dr. J. Son $\dagger$, J. Cha, Prof. J. Hong

Department of Materials Science and Engineering, Yonsei University

Seoul 03722, South Korea

E-mail: hong.jongill@yonsei.ac.kr

†Present address: Korea Institute of Science and Technology, Wanju 55324, South Korea 


\section{Supporting figures}

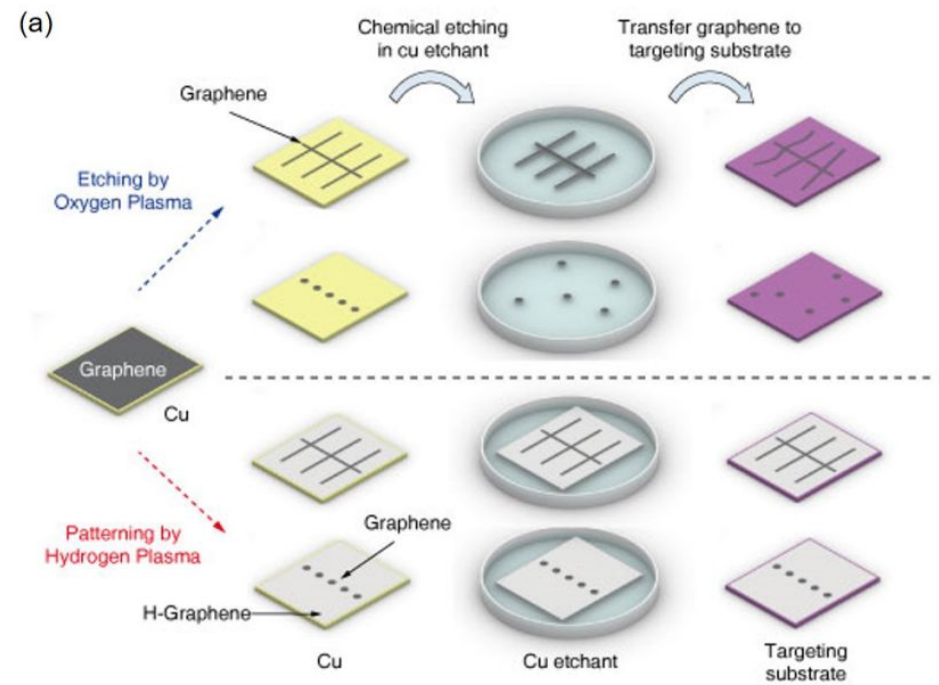

(b)

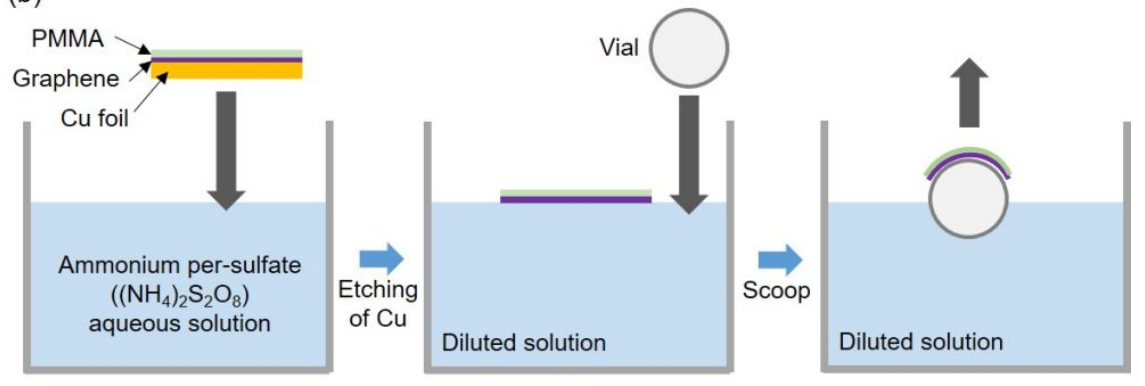

Figure S1. (a) A schematic comparison of the transferring process of graphene patterned by physical etching and hydrogenation, respectively. In conventional methods, complex patterns or island-like patterns are almost impossible to transfer because they float in the $\mathrm{Cu}$ etchant during the etching out of the $\mathrm{Cu}$ foil unless extra steps are added to maintain the pattern shapes before the transfer. Our direct transfer of graphene patterns on $\mathrm{Cu}$ foils after hydrogenation has an outstanding advantage over other methods since it makes it possible not only to transfer complex or island-like patterns to flexible substrates but also to reduce the number of steps of the patterning process. This is because hydrogenated graphene physically supports patterned graphene and vice versa. (b) A schematic illustrating the transfer method of graphene patterned by hydrogenation onto curved substrates. 


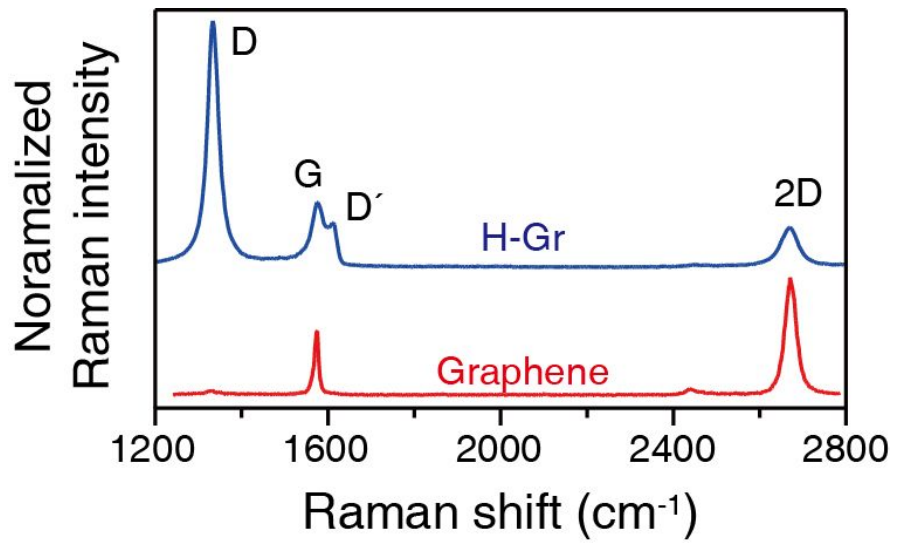

Figure S2. Raman signals of graphene and hydrogenated graphene (H-Gr). After hydrogenation, graphene has been transformed to H-Gr. The D-peak intensity is pronounced due to the formation of $s p^{3}$ bonding resulting from the chemisorbed hydrogen on carbon atoms. 


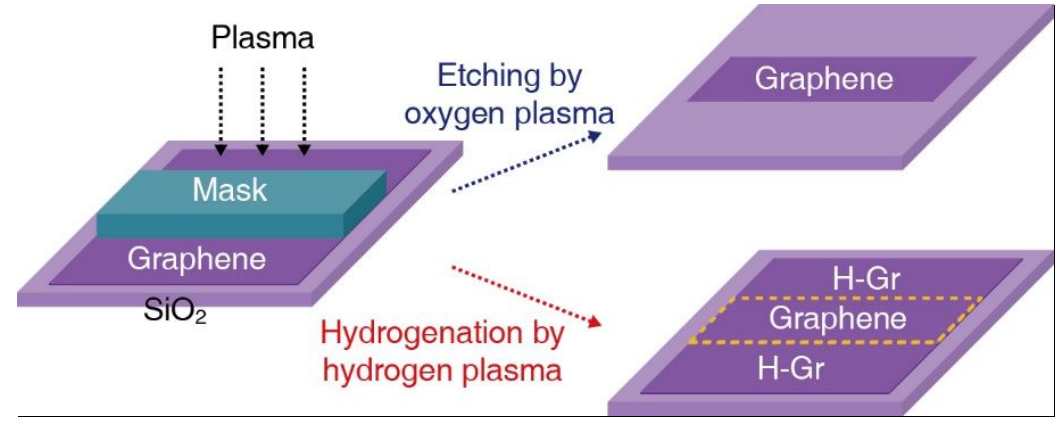

Figure S3. Schematic illustrating the two patterning methods: etching by oxygen plasma and hydrogenation by hydrogen plasma. 


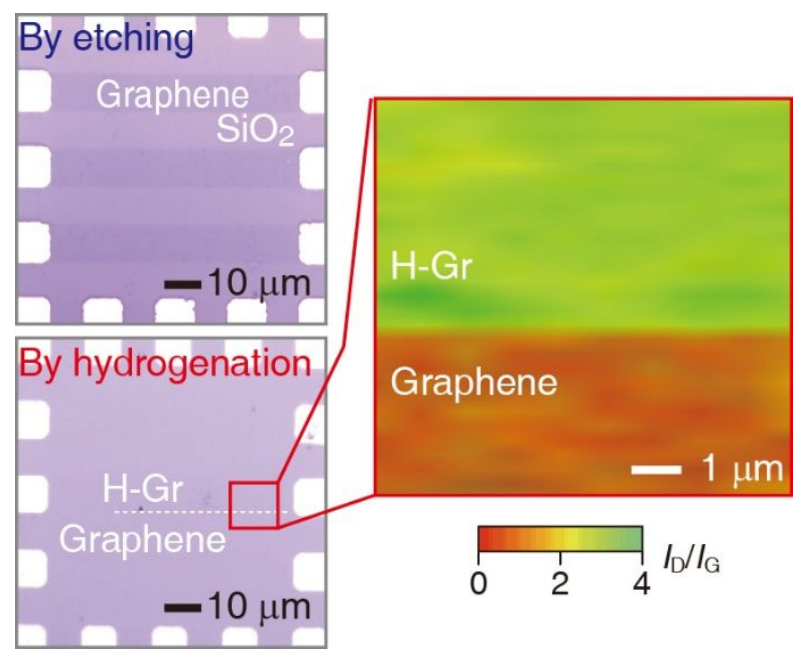

Figure S4. Optical microscopic images for graphene channels patterned by oxygen plasma and hydrogenation, respectively. The graphene channel and $\mathrm{H}-\mathrm{Gr}$ are optically indistinguishable but they are clearly identified in the Raman mapping of $I_{\mathrm{D}} / I_{\mathrm{G}}$. 


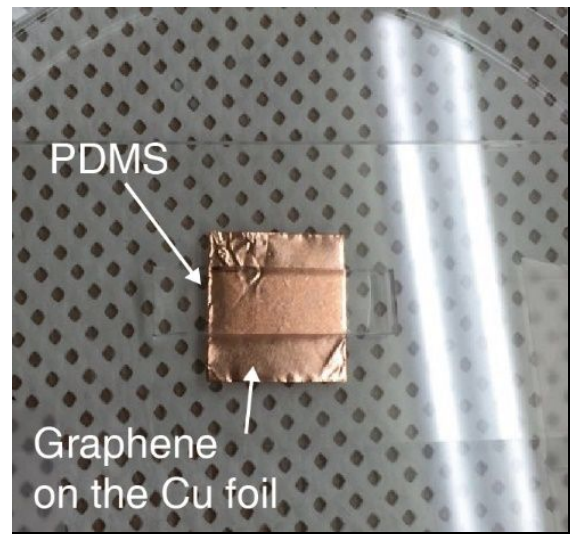

Figure S5. Optical image of graphene on the $\mathrm{Cu}$ foil before hydrogenation. The center of the $\mathrm{Cu}$ foil is protected with the PDMS mask, and the unprotected region is hydrogenated. 

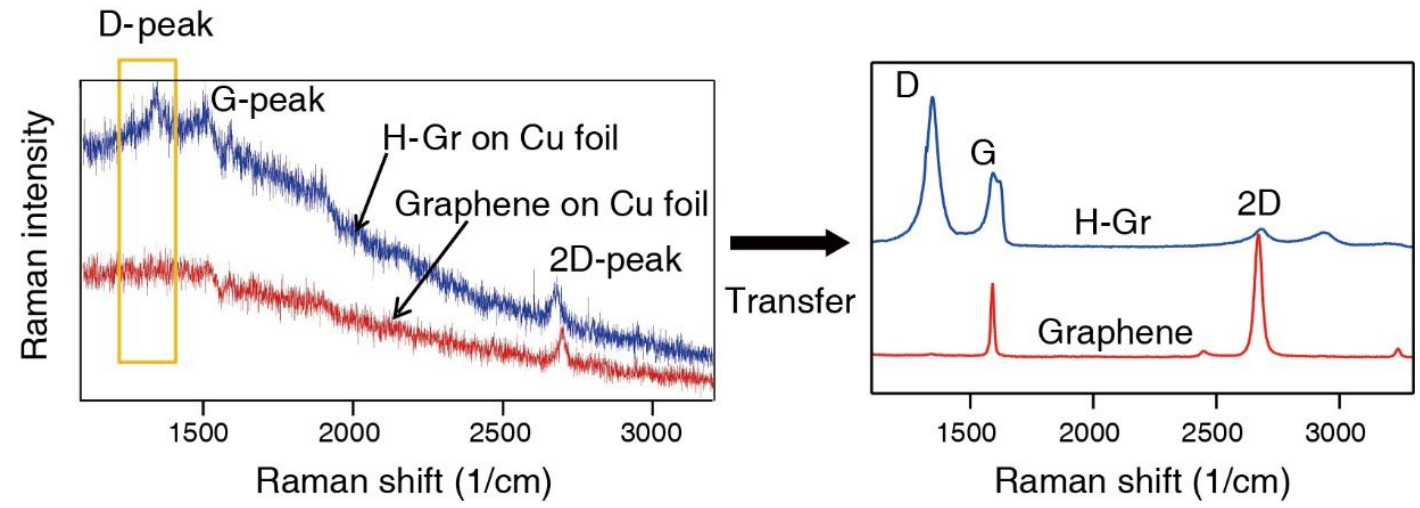

Figure S6. Raman spectra of graphene and $\mathrm{H}-\mathrm{Gr}$ on the $\mathrm{Cu}$ foil before and after transfer. Even after transfer, no chemical degradation or dehydrogenation is observed in $\mathrm{H}-\mathrm{Gr}$. 


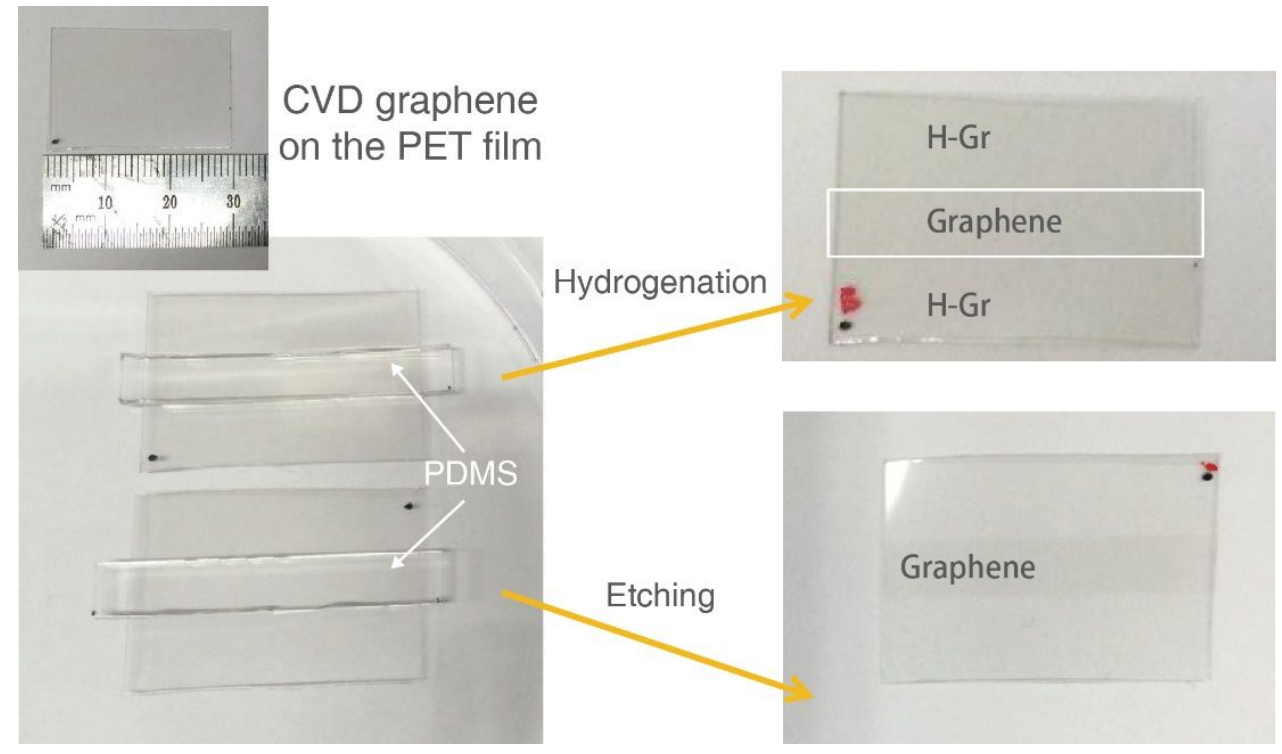

Figure S7. Optical images of CVD graphene on PET films: graphene patterned via hydrogenation (top right top) or via oxygen-plasma etching (bottom right). 